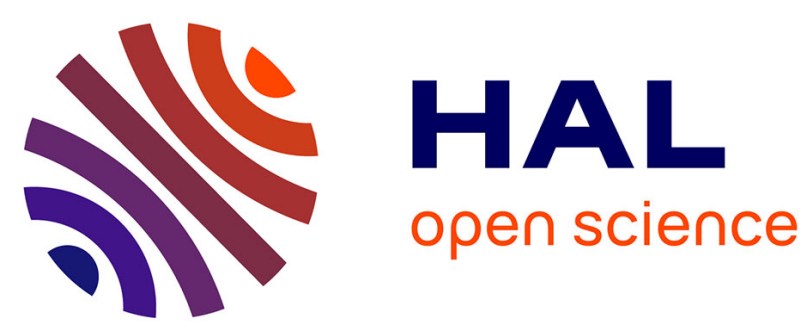

\title{
Ferroelectric $\mathrm{AgNa}(\mathrm{NO} 2) 2$ crystals as novel highly efficient nonlinear optical material: Phase matched second harmonic generation driven by a spontaneous and electric field induced polarizations
}

Bouchta Sahraoui, Robert Czaplicki, A. Klopperpieper, A.-S. Andrushchak, A.-V. Kityk

\section{To cite this version:}

Bouchta Sahraoui, Robert Czaplicki, A. Klopperpieper, A.-S. Andrushchak, A.-V. Kityk. Ferroelectric $\mathrm{AgNa}(\mathrm{NO} 2) 2$ crystals as novel highly efficient nonlinear optical material: Phase matched second harmonic generation driven by a spontaneous and electric field induced polarizations. Journal of Applied Physics, 2010, 107 (11), Non spécifié. 10.1063/1.3415545 . hal-03343401

\section{HAL Id: hal-03343401 \\ https://univ-angers.hal.science/hal-03343401}

Submitted on 14 Sep 2021

HAL is a multi-disciplinary open access archive for the deposit and dissemination of scientific research documents, whether they are published or not. The documents may come from teaching and research institutions in France or abroad, or from public or private research centers.
L'archive ouverte pluridisciplinaire HAL, est destinée au dépôt et à la diffusion de documents scientifiques de niveau recherche, publiés ou non, émanant des établissements d'enseignement et de recherche français ou étrangers, des laboratoires publics ou privés. 


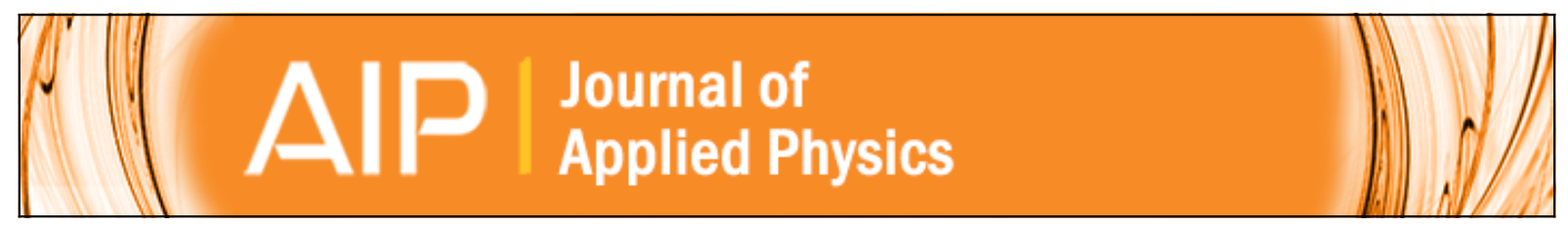

Ferroelectric AgNa ( NO 2 ) 2 crystals as novel highly efficient nonlinear optical material: Phase matched second harmonic generation driven by a spontaneous and electric field induced polarizations

B. Sahraoui, R. Czaplicki, A. Klöpperpieper, A. S. Andrushchak, and A. V. Kityk

Citation: Journal of Applied Physics 107, 113526 (2010); doi: 10.1063/1.3415545

View online: http://dx.doi.org/10.1063/1.3415545

View Table of Contents: http://scitation.aip.org/content/aip/journal/jap/107/11?ver=pdfcov

Published by the AIP Publishing

\section{Articles you may be interested in}

Multi-direction high-efficiency second harmonic generation in ellipse structure nonlinear photonic crystals Appl. Phys. Lett. 105, 151106 (2014); 10.1063/1.4898187

Large second-order optical nonlinearity in a ferroelectric molecular crystal of croconic acid with strong intermolecular hydrogen bonds

Appl. Phys. Lett. 102, 162901 (2013); 10.1063/1.4802727

Spontaneous and electric field induced quadratic optical nonlinearity in ferroelectric crystals AgNa ( NO 2 ) 2 Appl. Phys. Lett. 96, 061911 (2010); 10.1063/1.3315941

Broadband quasi-phase-matched second-harmonic generation in a nonlinear photonic crystal J. Appl. Phys. 100, 023120 (2006); 10.1063/1.2213150

Electric field induced second harmonic generation with and without fringes

Rev. Sci. Instrum. 71, 3490 (2000); 10.1063/1.1287629

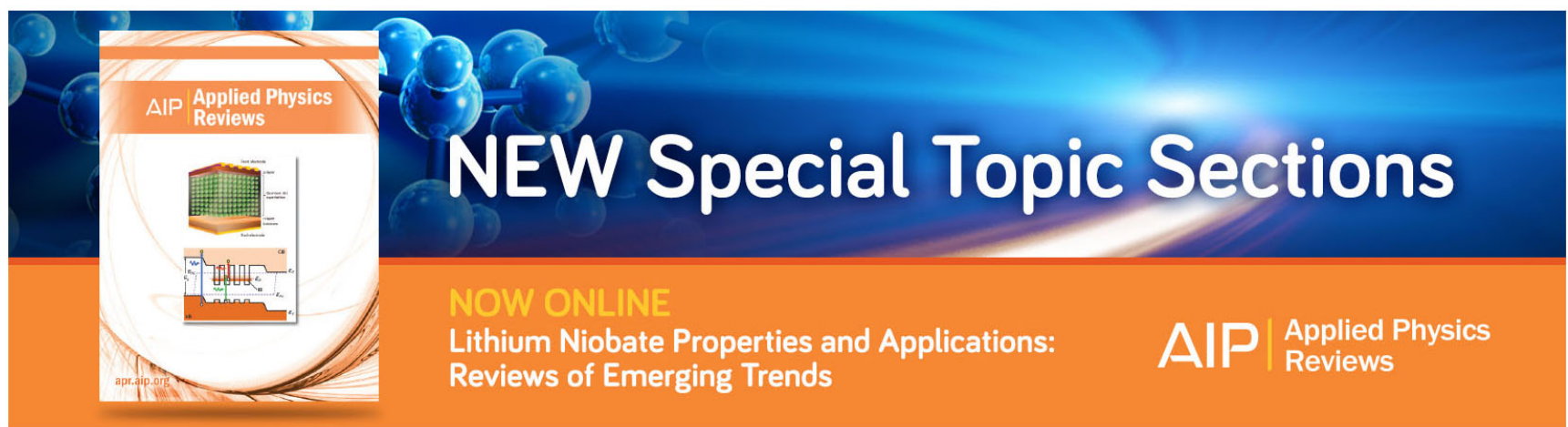




\title{
Ferroelectric $\mathrm{AgNa}\left(\mathrm{NO}_{2}\right)_{2}$ crystals as novel highly efficient nonlinear optical material: Phase matched second harmonic generation driven by a spontaneous and electric field induced polarizations
}

\author{
B. Sahraoui, ${ }^{1}$ R. Czaplicki, ${ }^{1}$ A. Klöpperpieper, ${ }^{2}$ A. S. Andrushchak, ${ }^{3}$ and A. V. Kityk ${ }^{4, a)}$ \\ ${ }^{1}$ Institut des Sciences et Technologies Moléculaires d'Angers, MOLTECH ANJOU-UMR CNRS 6200, \\ Université d'Angers, 2 bd Lavoisier, 49045 Angers Cedex 2, France \\ ${ }^{2}$ Technische Physik, Universität des Saarlandes, 66041 Saarbrücken, Germany \\ ${ }^{3}$ Lviv Polytechnic National University, 12 S. Bandera Str., 79013 Lviv, Ukraine \\ ${ }^{4}$ Faculty of Electrical Engineering, Czestochowa University of Technology, Al. Armii Krajowej 17, 42-200 \\ Czestochowa, Poland
}

(Received 7 February 2010; accepted 3 April 2010; published online 7 June 2010)

\begin{abstract}
Paper reports the second harmonic generation (SHG) in ferroelectric $\mathrm{AgNa}\left(\mathrm{NO}_{2}\right)_{2}$ crystals being driven by the spontaneous and electric field induced polarizations. Obtained results are interpreted within the phenomenological theory which considers the free energy describing the interaction between the spontaneous or electric field induced polarizations and spatially inhomogeneous electric polarizations resulted from propagating optical waves. Relatively high magnitudes of the effective second order nonlinear optical (NLO) susceptibilities in these crystals are combined with several phase matching geometries which allows to consider them as high-performance materials for potential NLO applications, such as parametric generation and amplification, frequency doubling, or other applications that require high-efficient frequency conversion. In addition, an anomalously large response of NLO susceptibilities with respect to an applied electric field has been found in the vicinity of the Curie point. This may also have a number of applications, especially in those devices where an efficient tunable control of SHG intensity is demanded. (C) 2010 American Institute of Physics. [doi:10.1063/1.3415545]
\end{abstract}

\section{INTRODUCTION}

Ferroelectric crystals are considered as very promising materials for a number of optoelectronic applications. Most of them are based on parametrical or nonlinear optical (NLO) phenomena such as electro-optic effect, second harmonic generation (SHG), parametric down conversion, parametric amplification or generation, etc., being widely used for high-efficient light modulation or its frequency conversion. ${ }^{1-3}$ The SHG, known also as frequency doubling, represents the phenomenon tightly coupled with nonlinear second order optical susceptibilities $\chi_{i j k}^{(2)}$ which are the components of third order polar tensor. Most of the ferroelectric materials are centrosymmetric in paraelectric phase, thus all the NLO susceptibilities $\chi_{i j k}^{(2)}=0$, i.e., the SHG is forbidden by the symmetry. Upon cooling a phase transition (PT) to the ferroelectric state breaks the symmetry leading to a dramatic change in a SHG efficiency. A spontaneous polarization $P_{s}$ plays, in such transformation, a role of symmetry breaking parameter. It describes quantitatively structural changes below the Curie point $T_{c}$ which result to a lowering of the crystal symmetry. Accordingly, the behavior of NLO susceptibilities $\chi_{i j k}^{(2)}$ in the ferroelectric phase correlate with the $P_{s}$ thereby the SHG may be considered here as an effect driven by the spontaneous polarization and, in analogy to the electro-optical terminology, ${ }^{4}$ one may call it as spontaneous $S H G .^{5}$

\footnotetext{
${ }^{a)}$ Author to whom correspondence should be addressed. Electronic mail: andriy.kityk@univie.ac.at.
}

Alternatively, the inversion center of the parent structure may be lost under an electric field applied to the crystal. This also results in SHG. Its magnitude is essentially related to the electric polarization of the media, thus it is proportional to the dielectric constant. For this reason the field induced $S H G$ is usually a weak effect as long as the classical linear dielectrics are considered. The situation changes cardinally if one deals with the proper ferroelectrics. Their dielectric constant along the polar axis behaves anomalously in the vicinity of the Curie point $T_{c}{ }^{6}$ Accordingly, the SHG, driven by an applied electric field may rise here even up to several orders of magnitude. This is of a considerable interest in a light of a large number of applications. In particular, by choosing a temperature range close to $T_{c}$ one may reach a considerable modulation efficiency and/or substantially reduce an operating voltage of corresponding optoelectronic devices. Other types of applications require tunable intensity of SHG signal which in the case of an optical cell made of a ferroelectric crystal material can be easily adjusted either by a temperature change and/or by an electric field tuning.

In this work, we report the phase matched SHG in ferroelectric $\mathrm{AgNa}\left(\mathrm{NO}_{2}\right)_{2}$ being driven by spontaneous and electric field induced polarizations. Actual paper represents considerable extensions of the data published recently in the letter article. ${ }^{5}$ The $\operatorname{AgNa}\left(\mathrm{NO}_{2}\right)_{2}$ crystals exhibit the PT at $T_{c} \approx 38{ }^{\circ} \mathrm{C}$ (Refs. 7 and 8) from a paraelectric phase (space group $\mathrm{D}_{2 h}^{24}$ ) to the proper ferroelectric phase (space group $\mathrm{C}_{2 v}^{19}$ ). The dielectric, ${ }^{7-9}$ specific-heat, ${ }^{10}$ and elastic measurements ${ }^{11}$ have revealed that the PT is of the first order, however very close to the second order one. For this 
reason the anomalies of dielectric and thermal properties can be well explained by a Landau theory taking into account the near tricritical character of this PT. As a consequence the leading term in the free energy expansion is of the sixth order in the polarization $P$. Ferroelectricity in $\mathrm{AgNa}\left(\mathrm{NO}_{2}\right)_{2}$ appears due to an ordering of the $\mathrm{NO}_{2}{ }^{-}$dipoles ${ }^{12}$ which at room temperature give quite large spontaneous polarization (about $8 \mu \mathrm{C} / \mathrm{cm}^{2}$ ) being oriented along [010] crystallographic direction. ${ }^{8}$ The uniaxial ferroelectric $\mathrm{AgNa}\left(\mathrm{NO}_{2}\right)_{2}$ constitutes an exception in the sense of its polarization dynamics which is of Debye-type and appears in the kilohertz region. $9,13,14$

\section{BRIEF PHENOMENOLOGICAL ANALYSIS OF THE QUADRATIC OPTICAL NONLINEARITY IN $\operatorname{AGNA}\left(\mathrm{NO}_{2}\right)_{2}$}

In this section, we present a brief analysis of the second order optical nonlinearity in ferroelectric $\mathrm{AgNa}\left(\mathrm{NO}_{2}\right)_{2}$. It is based on the phenomenological theory and allows to understand a relation between the NLO susceptibilities $\chi_{i j k}^{(2)}$ and the basic macroscopic characteristics of this ferroelectric material, i.e., its spontaneous polarization $P_{s}$ and/or dielectric susceptibility. We start from the general characterization of the ferroelectric PT in $\mathrm{AgNa}\left(\mathrm{NO}_{2}\right)_{2}$ crystals. Following the actual symmetry transformation $\left(\mathrm{D}_{2 h} \rightarrow \mathrm{C}_{2 v}\right)$ the Landau free energy may be written as: ${ }^{11}$

$$
F_{P}=\frac{1}{2} A\left(T-T_{0}\right) P^{2}+\frac{1}{4} B P^{4}+\frac{1}{6} C P^{6}-P E,
$$

where $P$ is the $Y$-component of the polarization being the order parameter of this model, $A, B$, and $C$ are the free energy expansion coefficients, $T_{0}$ is the Curie-Weiss temperature, and $E$ is the electric field. Specific of the paraelectricto-ferroelectric PT in $\mathrm{AgNa}\left(\mathrm{NO}_{2}\right)_{2}$ causes several requirements in regard to the expansion coefficients. We indeed deal with a weakly first order transition being very close to the tricritical point. Such formulation is equivalent to the case when the coefficient $B$ is represented by small negative value whereas the two other coefficients $A$ and $C$ are essentially positive. Considering the case when $E=0$ the actual PT temperature $T_{c}$ and the equilibrium value of the spontaneous polarization $P_{S}$ may be obtained in usual way by solving simultaneously a set of the two following equations:

$$
F_{P}=0, \quad \partial F_{P} / \partial P=0
$$

which yields:

$$
T_{c}=T_{0}+3 B^{2} / 16 A C
$$

and

$$
\begin{aligned}
& P_{s}=0\left(T>T_{c}\right), \\
& P_{s}^{2}=\left(-B+\sqrt{B^{2}-4 C A\left(T-T_{0}\right)}\right) / 2 C\left(T \leq T_{c}\right) .
\end{aligned}
$$

Figure 1(a) shows schematically the $T$-dependence of $P_{s}(E$ $=0$, see solid line of blue color) in the vicinity of the Curie point $T_{c}$. If the electric field is applied $(E \neq 0)$ the equilibrium polarization $P^{+}=P_{s}+P_{\text {ind }}$ ( $P_{\text {ind }}$ is the field induced polarization) may be obtained by solving the electric equation

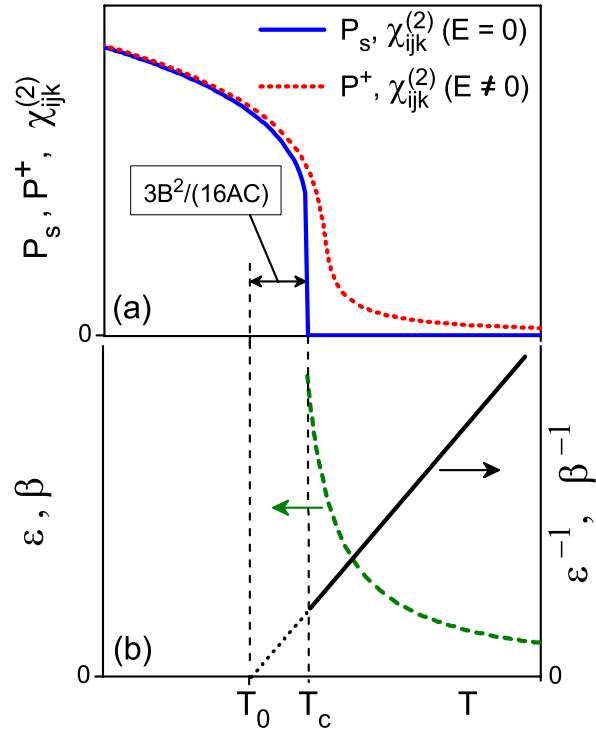

FIG. 1. (Color online) Temperature behavior of $P_{s}, P^{+}$, and $\chi_{i j k}^{(2)}$ (a); $\varepsilon, \beta$, $\varepsilon^{-1}$, and $\beta^{-1}$ vs $T$ (b) near the paraelectric-to-ferroelectric PT (schematic presentation).

of state $\left(\partial F_{P} / \partial P=-E\right)$. This leads to the dependence $P^{+}$versus $T$ as qualitatively shown by the dashed red line in Fig. 1(a). It should be emphasized that the crystal symmetry is different on both sides of the PT point only when $E=0$. For $\mathrm{AgNa}\left(\mathrm{NO}_{2}\right)_{2}$ it is centrosymmetric $\left(\mathrm{D}_{2 h}\right)$ above $T_{c}$ and noncentrosymmetric $\left(\mathrm{C}_{2 v}\right)$ below it. If $E \neq 0$ the symmetry is identical on both side of the Curie point $\left(\mathrm{C}_{2 v}\right)$ thus the PT loses it classical meaning because there is no break of the symmetry anymore here. Nevertheless, even at $E \neq 0$ most of physical properties keep its anomalous behavior in the vicinity of Curie point. One of such characteristics, being important for the further analysis of NLO properties, is the static dielectric susceptibility $\chi=\varepsilon_{0}^{-1} \partial P / \partial E$. Its inverse magnitude may be obtained by differentiating the electric equation of state

$$
\left(\varepsilon_{0} \chi\right)^{-1}=\partial E / \partial P=A\left(T-T_{0}\right)+3 B P^{2}+5 C P^{4} .
$$

Above $T_{c}$ and at small electric field $(E \rightarrow 0)$ the induced polarization $P_{\text {ind }} \equiv P^{+}$can be ignored in Eq. (5) (since $P^{+} \rightarrow 0$ ), accordingly, the static dielectric constant $\varepsilon=1+\chi \approx C_{0} /(T$ $\left.-T_{0}\right)$, where $C_{0}=\left(A \varepsilon_{0}\right)^{-1}$ is the Curie-Weiss constant. $\varepsilon$ and $\varepsilon^{-1}$ versus $T$ are shown schematically in Fig. 1(b). Obviously, the strength of dielectric anomaly near the Curie point $T_{c}$ is a matter of its closeness to the tricritical point at which the difference $\left(T_{c}-T_{0}\right) \rightarrow 0$ and $\varepsilon\left(T_{c}\right) \rightarrow \infty . \operatorname{AgNa}\left(\mathrm{NO}_{2}\right)_{2}$ in fact constitutes an example of the crystal with a weakly first order paraelectric-to-ferroelectric PT. For this reason the static dielectric constant indeed rises in the vicinity $T_{c}$ on about of the two orders of magnitude. ${ }^{7-9}$

To interpret the NLO properties we introduce into the free energy the electric polarizations induced by a propagating electromagnetic light waves. The approach offered here is in agreement with the Muller's formalism developed in Ref. 15. However, it is more general since we deal with the inhomogeneous free energy instead of the free energy in a local approximation as presented in Ref. 15. In some respect the forthcoming approach is based on principles similar to 
those used in the description of incommensurate phases (see, e.g., Refs. 16-19). The part of the free energy density which is responsible for the $\mathrm{SHG}$ in ferroelectric phase of $\mathrm{AgNa}\left(\mathrm{NO}_{2}\right)_{2}$ may be written as:

$$
\begin{aligned}
f_{\text {int }}(r, t)= & -\alpha_{2 j k l}^{0, \omega, \omega, 2 \omega}\left[P_{2}(0) P_{j}\left(\omega, k_{1}\right) P_{k}\left(\omega, k_{2}\right) P_{l}^{*}\left(2 \omega, k_{3}\right)\right. \\
& +c . c .]+\frac{1}{2 \varepsilon_{0} \chi_{i i}^{\Omega}} P_{i}(\Omega, K) P_{i}^{*}(\Omega, K),
\end{aligned}
$$

where $\varepsilon_{0}$ is the vacuum permittivity; $\chi_{i i}^{\Omega}$ is linear dielectric susceptibility at the frequency $\Omega \equiv \omega$ or $2 \omega ; \alpha_{2 j k l}^{0, \omega, \omega, 2 \omega}$ are the components of the fourth order polar tensor being invariant with respect to the symmetry operation of the point group $\mathrm{D}_{2 h}$ of the paraelectric phase; $P_{2}(0)$ is the $Y$-component of static spatially homogeneous polarization; and $P_{j}\left(\omega, k_{1}\right)$, $P_{k}\left(\omega, k_{2}\right)$, and $P_{l}\left(2 \omega, k_{3}\right)$ are the traveling polarization waves which can be presented as follows:

$$
\begin{aligned}
& P_{j}\left(\omega, k_{1}\right)=P_{j}\left(k_{1}\right) \exp (i \omega t), \\
& P_{k}\left(\omega, k_{2}\right)=P_{k}\left(k_{2}\right) \exp (i \omega t), \\
& P_{l}\left(\omega, k_{3}\right)=P_{l}\left(k_{3}\right) \exp (i 2 \omega t),
\end{aligned}
$$

with the complex amplitudes given as:

$$
\begin{aligned}
& P_{j}\left(k_{1}\right)=\left|P_{j}\left(k_{1}\right)\right| \exp \left(i k_{1} r\right), \\
& P_{k}\left(k_{2}\right)=\left|P_{k}\left(k_{2}\right)\right| \exp \left(i k_{2} r\right), \\
& P_{l}\left(k_{3}\right)=\left|P_{l}\left(k_{3}\right)\right| \exp \left(i k_{3} r\right) .
\end{aligned}
$$

The coupling $\alpha$-term in fact describes the NLO conversion process, i.e., the interaction between the homogeneous polarization $P_{2}(0)$, spatially inhomogeneous polarizations $P_{j}\left(\omega, k_{1}\right)$ and $P_{k}\left(\omega, k_{2}\right)$, induced by incident laser light and oscillating at the fundamental frequency $\omega$, and its second temporal harmonics $P_{l}\left(2 \omega, k_{3}\right)$. One may realize that the temporal variable in Eq. (6) has been added only formally. Indeed the free energy density appears to be time independent as it follows after inserting Eq. (7) into Eq. (6). One then obtains the following:

$$
\begin{aligned}
f_{\text {int }}(r)= & -\alpha_{2 j k l}^{0, \omega, \omega, \omega \omega}\left[P_{2}(0) P_{j}\left(k_{1}\right) P_{k}\left(k_{2}\right) P_{l}^{*}\left(k_{3}\right)+c . c .\right] \\
& +\frac{1}{2 \varepsilon_{0} \chi_{i i}^{\Omega}} P_{i}(K) P_{i}^{*}(K) .
\end{aligned}
$$

This expression is similar to the one used in Ref. 5. The difference is that the Eq. (1) of Ref. 5 is written for the particular case when $k_{1}=k_{2}=k_{3} / 2$, i.e., the birefringent phase matching between the interacting modes has been assumed there from the beginning. The free energy density Eq. (9) gives therefore a more general description of the problem. Inserting Eq. (8) into Eq. (9) leads to

$$
\begin{aligned}
f_{\text {int }}(r)= & -2 \alpha_{2 j k l}^{0, \omega, \omega, 2 \omega} P_{2}(0)\left|P_{j}\left(k_{1}\right)\left\|P_{k}\left(k_{2}\right)\right\| P_{l}\left(k_{3}\right)\right| \cos (\Delta k r) \\
& +\frac{1}{2 \varepsilon_{0} \chi_{i i}^{\Omega}}\left|P_{i}(K)\right|^{2},
\end{aligned}
$$

where $\Delta k=k_{1}+k_{2}-k_{3}$ is the mismatch wavenumber. The free energy of the NLO interaction corresponding to the active path length $L$ of the crystal may be obtained by spatial averaging of Eq. (10) which yields:

$$
\begin{aligned}
F_{\text {int }}= & \frac{1}{L} \int_{-L / 2}^{L / 2} f_{\text {int }}(r) d r=-2 \alpha_{2 j k l}^{0, \omega, \omega, 2 \omega} P_{2}(0)\left|P_{j}\left(k_{1}\right)\right| \\
& \times\left|P_{k}\left(k_{2}\right)\right|\left|P_{l}\left(k_{3}\right)\right| \operatorname{sinc}(\Delta k L / 2)+\frac{1}{2 \varepsilon_{0} \chi_{i i}^{\Omega}}\left|P_{i}(K)\right|^{2} .
\end{aligned}
$$

It must be emphasized that the free energy Eq. (11) is indeed obtained ignoring the spatial variation in the real amplitudes $\left|P_{j}\left(k_{1}\right)\right|$ and $\left|P_{k}\left(k_{2}\right)\right|$ which returns us to a local approximation. The amplitude of the polarization wave with doubled frequency $\left|P_{l}\left(k_{3}\right)\right|$ can be derived by minimization of the free energy $\left(\partial F_{\text {int }} / \partial\left|P_{l}\left(k_{3}\right)\right|=0\right)$, which leads to:

$$
\begin{aligned}
\left|P_{l}\left(k_{3}\right)\right|= & 2 \alpha_{2 j k l}^{0, \omega \omega, \omega \omega} \varepsilon_{0} \chi_{l l}^{2 \omega} P_{2}(0)\left|P_{j}\left(k_{1}\right)\right| \\
& \times\left|P_{k}\left(k_{2}\right)\right| \operatorname{sinc}(\Delta k L / 2) .
\end{aligned}
$$

If the phase matching may be achieved $(\Delta k=0)$, which indeed is the case of $\mathrm{AgNa}\left(\mathrm{NO}_{2}\right)_{2}$ crystals, the sinc-factor in Eq. (11) equals 1 . This equation then coincides with the one given in Ref. 5. Using the macroscopic relations $\left|P_{j}\left(k_{1}\right)\right|$ $=\varepsilon_{0} \chi_{j j}^{\omega}\left|E_{j}(\omega)\right|$ and $\left|P_{k}\left(k_{2}\right)\right|=\varepsilon_{0} \chi_{k k}^{\omega}\left|E_{k}(\omega)\right|$ one gets the second order NLO susceptibility as follows:

$$
\chi_{l j k}^{(2)}=2 \varepsilon_{0}^{2} \alpha_{2 j k l}^{0, \omega, \omega, 2 \omega} P_{2}(0) \chi_{l l}^{2 \omega} \chi_{j j}^{\omega} \chi_{k k}^{\omega} .
$$

This equation is indeed equivalent to the Eq. (4) of Ref. 15 taking into account that its third order tensor components $\delta_{j k l} \equiv 2 \alpha_{2 j k l}^{0, \omega, \omega, 2 \omega} P_{2}(0)$. Above $T_{c}$ and at $E=0$ the static polarization $P_{2}(0) \equiv P_{s}=0$ thus all the $\delta_{j k l}=0$ as required by centrosymmetricity of the paraelectric phase.

If $E=0$ then $P_{2}(0) \equiv P_{s}$. In the paraelectric phase $(T$ $\left.>T_{c}\right) P_{s}=0$ thereby all the components $\chi_{l j k}^{(2)}=0$, and the SHG is absent. In the ferroelectric phase $\left(T \leq T_{c}\right) \chi_{l j k}^{(2)} \propto P_{s}$. Both $\chi_{l j k}^{(2)}$ and $P_{s}$ are expected to exhibit an identical temperature behavior [see solid blue curve in Fig. 1(a)], i.e., the SHG is driven here by spontaneous polarization only.

In the case when an external electric field is applied along the polar axis $(E \| Y) P_{2}(0) \equiv P^{+}$. Accordingly, the SHG is expected to be observed both above and below $T_{c}$ whereas $\chi_{l j k}^{(2)} \propto P^{+}$as it is shown schematically in the Fig. 1(a) [see dashed red curve]. Especially interesting is the temperature range above $T_{c}$. Here $P^{+}$is defined exclusively by the electric field induced polarization $P_{\text {ind }}$ having anomalous increase in the vicinity of $T_{c}$. Since $P_{\text {ind }}=\varepsilon_{0}(\varepsilon-1) E$ the NLO constant $\beta$, defined as $d \chi_{l j k}^{(2)} / d E$, may be expressed as follows:

$$
\beta=2 \varepsilon_{0}^{3}(\varepsilon-1) \alpha_{2 j k l}^{0, \omega, \omega, 2 \omega} \chi_{l l}^{2 \omega} \chi_{j j}^{\omega} \chi_{k k}^{\omega} .
$$

In the proper ferroelectrics $\varepsilon \gg 1$, thus the temperature variations $\beta(T)$ and $\varepsilon(T)$ are expected to be identical. In the vicinity of $T_{c}$ they anomalously rise as schematically shown in Fig. 1(b).

\section{EXPERIMENTAL}

Single crystals of $\mathrm{AgNa}\left(\mathrm{NO}_{2}\right)_{2}$ were grown from aqueous solution containing 9.8 wt $\% \mathrm{AgNO}_{2}$ and $37.2 \mathrm{wt} \%$ $\mathrm{NaNO}_{2}$ by the slow evaporation method at constant tempera- 


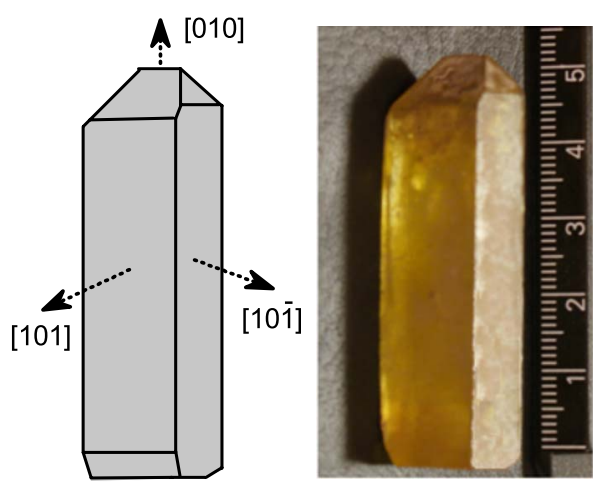

FIG. 2. (Color online) Habit of $\mathrm{AgNa}\left(\mathrm{NO}_{2}\right)_{2}$ crystal and its crystallographic orientation.

ture $\left(\sim 25^{\circ} \mathrm{C}\right)$. Figure 2 shows the habit of the grown single crystals and its crystallographical orientation. We used the standard crystallographic axes set for the paraelectric phase; $a=8.05 \AA, \quad b=10.77 \AA$, and $c=10.76 \AA .^{20}$ Crystals $\mathrm{AgNa}\left(\mathrm{NO}_{2}\right)_{2}$ are yellowish with the perfect cleavage plane perpendicular to [101] and [101] -directions. Accordingly, the geometry of samples was adjusted to these planes, i.e., we used the slabs or plates having perfectly cleaved faces [101] $(\text { or }[10 \overline{1}])^{7}$ without their further polishing. The silver paste electrodes were deposited on conventionally polished [010]faces.

The SHG was excited by means of nanosecond IR Q-switched laser Vector 1064-3000-30 ( $\lambda=1064 \mathrm{~nm})$ and registered in a standard setup described earlier in Ref. 21. The determination of the NLO coefficients associated with SHG was performed by the Maker fringe technique ${ }^{22}$ as described in detail by Jerphagnon and Kurtz. ${ }^{23}$ The halfwave retarder and Glan-Thomson polarizer was used to set polarization (horizontal or vertical) of the incident laser beam depending on phase matching geometry (PMG) being chosen in the experiment. The samples were set into thermostabilized optical cell operating by a temperature controller with an accuracy of the temperature stabilization of about $0.01{ }^{\circ} \mathrm{C}$. The thermostabilized cell was attached to an optical table in which the sample has been rotated by means of a step motor with angular step of $0.01^{\circ}$ and rotation speed 1-5 $\mathrm{deg} / \mathrm{min}$. The outgoing SHG light was filtered by means of interference filter(s) $(\lambda=532 \mathrm{~nm}, \Delta \lambda=4 \mathrm{~nm})$ and registered by photomultiplier. Consequently, the signal was detected using a box car integrator and then recorded.

\section{RESULTS AND DISCUSSION}

In the paraelectric phase of $\mathrm{AgNa}\left(\mathrm{NO}_{2}\right)_{2}$ crystals the SHG is symmetry forbidden. In the ferroelectric phase it is allowed by the symmetry, however these crystals exhibit only a very weak intense SHG signal if the light propagates

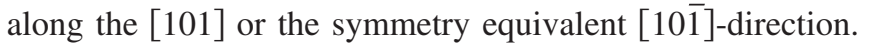
We explain this by a lack of the phase matching in NLO interaction along these crystallographic directions. Nevertheless, the phase matching indeed can be achieved in slightly tilted geometries. The insets in Fig. 3 demonstrate the two examples of such PMGs called hereafter as PMG-I and PMG-II, respectively. The PMG-I is defined by two angles
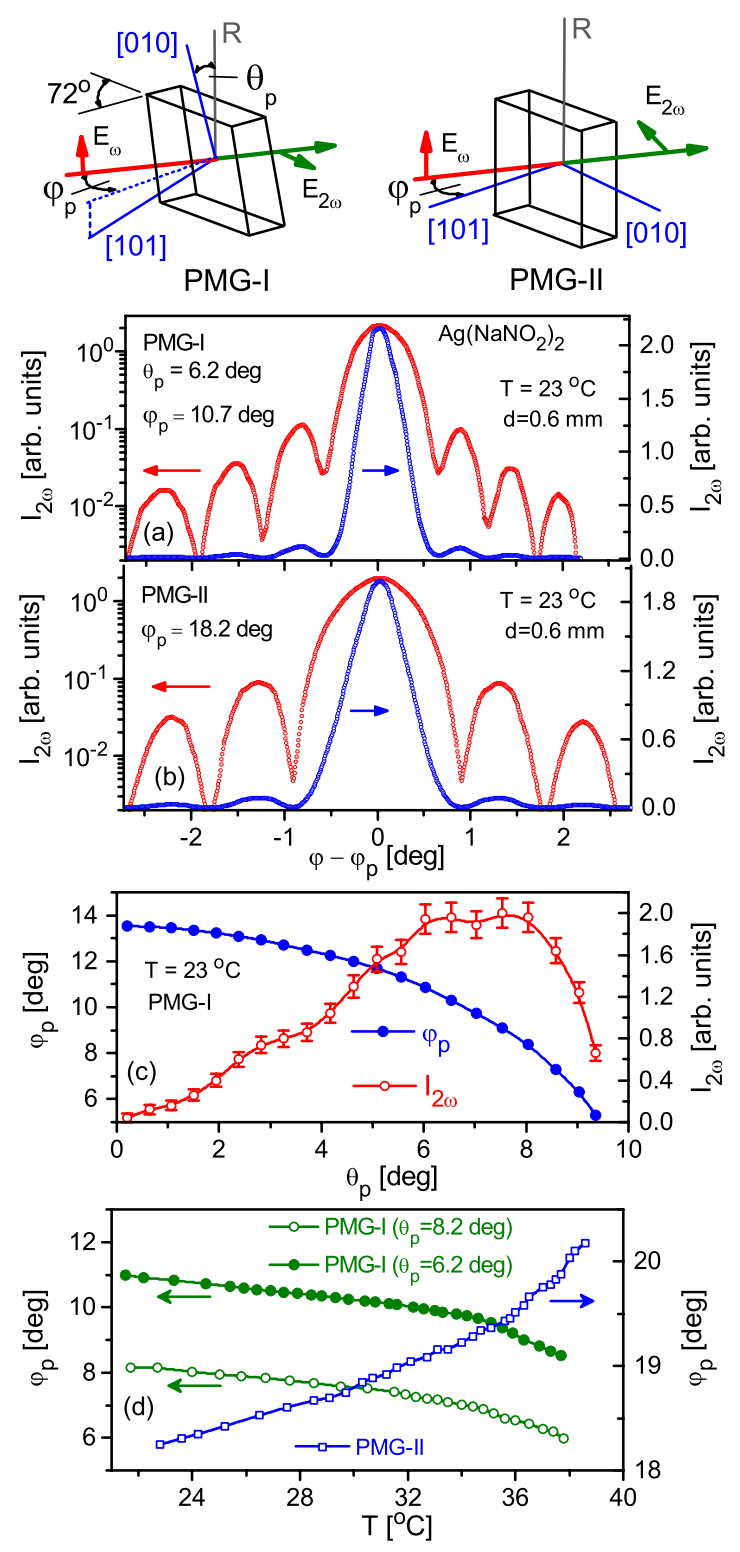

FIG. 3. (Color online) Phase matching in $\mathrm{AgNa}\left(\mathrm{NO}_{2}\right)_{2}$ crystals. The insets show two examples of PMGs (PMG-I and PMG-II). Panels (a) and (b) show the angular dependencies of the SHG intensity $I_{2 \omega}$ in the vicinity of the phase matching angle $\varphi_{p}$ at $T=23{ }^{\circ} \mathrm{C}$ for PMG-I and PMG-II, respectively. (c) $\theta_{p}$-dependence of $\varphi_{p}$ and $I_{2 \omega}$ in PMG-I at $T=23{ }^{\circ} \mathrm{C}$. (d) Temperature dependences of $\varphi_{p}$ at fixed and $\theta_{p}$ in PMG-I and PMG-II.

$\theta_{p}$ and $\varphi_{p}$. The incident intense nanosecond-pulsed light $(\lambda$ $=1064 \mathrm{~nm}, \tau=300 \mathrm{ps}$ ) is polarized vertically whereas the outcoming SHG light $(\lambda=532 \mathrm{~nm})$ exhibits nearly horizontal polarization at small $\theta_{p}$. The angle $\varphi_{p}$ depends on $\theta_{p}$ as shown in Fig. 3(c) whereas SHG intensity $I_{2 \omega}$ passes through its maximum magnitudes at $\theta_{p} \approx 6^{\circ}-8^{\circ}$. For the PMG-II the rotation $R$-axis is set perpendicularly to the [101] and [010]directions. The phase matching direction is defined here by symmetry equivalent angles $\pm \varphi_{p}\left(\theta_{p}=0\right)$, incident laser beam is polarized vertically whereas outgoing intense SHG exhibits polarization turned on of angle about $\pm 52^{\circ}\left(T=23^{\circ} \mathrm{C}\right)$. In both geometries, the $\varphi$-dependence of SHG intensity $I_{2 \omega}$ reveals Maker-fringe pattern [see Figs. 3(a) and 3(b)] which in a close vicinity of $\varphi_{p}$ roughly scales with $\propto L^{2}\left(\chi_{\text {eff }}^{(2)}\right)^{2} \operatorname{sinc}^{2}\left[a\left(\varphi-\varphi_{p}\right) L / 2\right]$, where $L$ and $\chi_{\text {eff }}^{(2)}$ are the path 


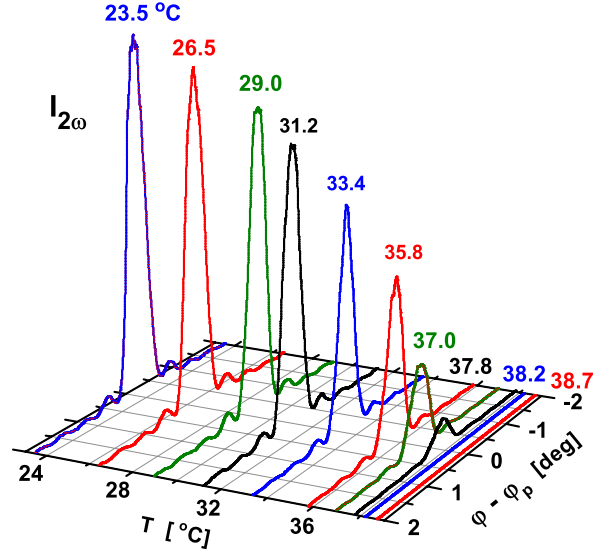

FIG. 4. (Color online) The angular $\varphi$-dependences of the SHG intensity $I_{2 \omega}$ in the vicinity of the phase matching angle $\varphi_{p}\left(\theta=\theta_{p}\right)$ at different temperatures. The maximum of $I_{2 \omega}$ corresponds to PMG-I defined by the angles $\varphi_{p}$ and $\theta_{p}$.

length of NLO interaction and the effective second order NLO susceptibility, respectively, coefficient $a$ define the angular dependence of the mismatch vector $\Delta k$ in linear approximation $\left[\Delta k=a\left(\varphi-\varphi_{p}\right)\right]$. A challenging question is a type of the observed phase matching, i.e., whether it is of birefringent origin or one deals with so-called quasiphase matching caused by eventual ferroelectric domain structure. An exact answer on this question could be obtained having measured the principal refractive indices at $\lambda=532$ and 1064 $\mathrm{nm}$. Unfortunately, the refractometry measurements appear to be really problematic along the principal crystallographic directions of $\mathrm{AgNa}\left(\mathrm{NO}_{2}\right)_{2}$ crystals. Due to a perfect splitting of the single crystals (parallel to [101] and/or [101] cleavage planes) we were not able to prepare crystal cuts of sufficiently good optical quality along other crystallographic directions, in particular, the cuts perpendicular to the principal crystallographic directions which are required for eventual interferometric and/or refractometric measurements. On the other hand, it must be emphasized that in our case the measurements were performed on fresh samples being cut from the crystals that were grown in the ferroelectric phase. The obtained in such way crystals are usually single domain. The results were reproduced on several samples. Also the electric field up to $3 \mathrm{kV} / \mathrm{cm}$ applied along the polar axis did not change the SHG signal. For these reasons we believe that one deals with the birefringent phase matching rather than with the quasiphase one provided by a regular ferroelectric domain structure.

Figure 4 shows the angular dependences of the SHG intensity $I_{2 \omega}$ of $\mathrm{AgNa}\left(\mathrm{NO}_{2}\right)_{2}$ crystals in the vicinity of the phase matching angle $\varphi_{p}$ being recorded at different temperatures. The maximum of $I_{2 \omega}(\phi)$ corresponds here to PMG-I defined by angles $\varphi_{p}$ and $\theta_{p}$. One can see that the character of the Maker fringe patterns itself does not change significantly while the temperature rises. Only the SHG signal significantly decreases by approaching the ferroelectric-toparaelectric PT and completely vanishes at $T \geq T_{c}$.

In accordance with the point group $\mathrm{C}_{2 v}$ the effective susceptibility $\chi_{\text {eff }}^{(2)}$ in ferroelectric phase is expressed through the partial contributions of NLO tensor components $\chi_{222}^{(2)}, \chi_{233}^{(2)}$,
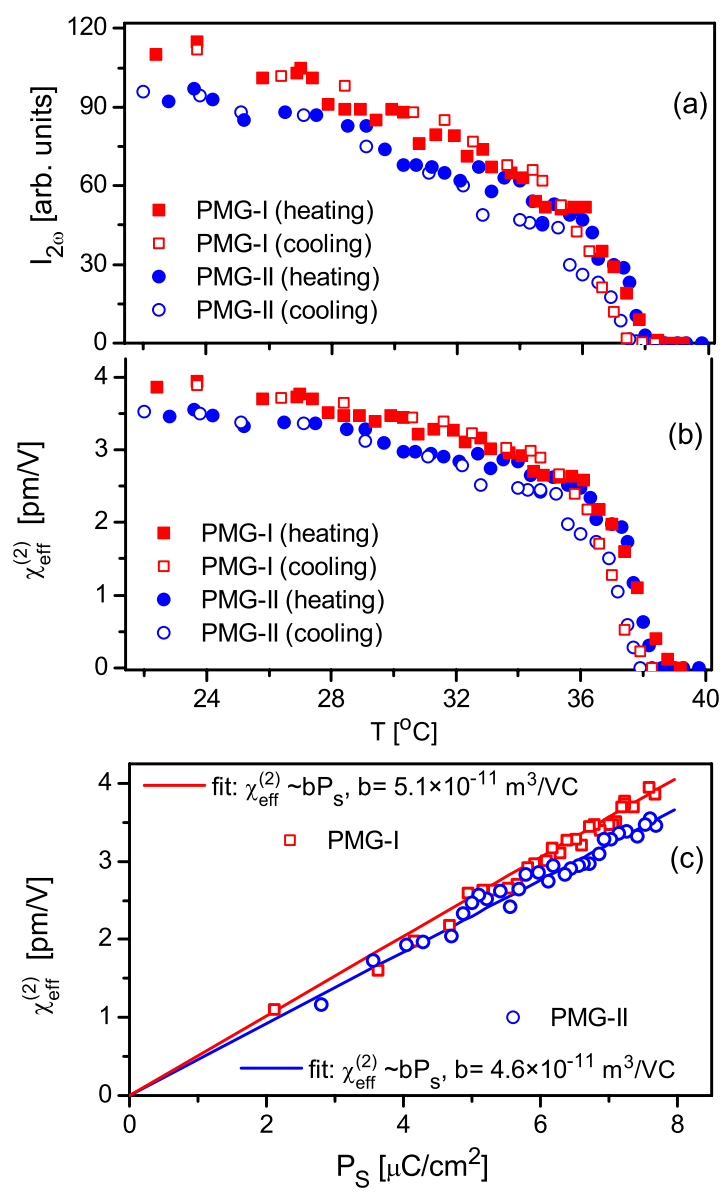

FIG. 5. (Color online) SHG in ferroelectric $\mathrm{AgNa}\left(\mathrm{NO}_{2}\right)_{2}$ crystals. (a) Temperature dependences of the SHG intensity $I_{2 \omega}$ measured in PMG-I and PMG-II at heating and cooling. (b) Effective NLO susceptibility $\chi_{\text {eff }}^{(2)}$ vs $T$ determined in the reference measurements $\left[I_{2 \omega}(T) / I_{2 \omega}^{\mathrm{SiO}_{2}}\left(\chi_{111}\right)\right]$ at heating and cooling in PMG-I (solid and open squares) and PMG-II (solid and open circles), respectively. (c) $\chi_{\mathrm{eff}}^{(2)}$ vs the spontaneous polarization $P_{s}$ in PMG-I and PMG-II at heating $\left[P_{s}(T)\right.$-dependence has been taken from Ref. 8]. Solid lines are the best linear fits of $\chi_{\mathrm{eff}}^{(2)}\left(P_{s}\right)$. Their slopes define the magnitudes of the coupling constant $b$.

$\chi_{211}^{(2)}, \chi_{121}^{(2)}$, and $\chi_{323}^{(2)}$ each of which is proportional to the spontaneous polarization $P_{s}$ as has been shown in Sec. II. On the other hand, the path length $L$ and the effective susceptibility $\chi_{\text {eff }}^{(2)}$ are both in principle dependent on the angles $\theta$ and $\varphi$. However at fixed $\theta=\theta_{p}$ the other phase matching angle $\varphi_{p}$, corresponding to the maximum of SHG intensity, is only very weakly temperature dependent in the ferroelectric phase for both PMGs [see Fig. 3(d)]. Basing on these facts our analysis shows that the weight factors bonded to the partial contributions of $\chi_{i j k}^{(2)}$ into $\chi_{\text {eff }}^{(2)}$, either do not depend at all or change only very slightly with the temperature due to a weakly varying $\varphi_{p}(T)$-dependence. Accordingly, $\chi_{\text {eff }}^{(2)}(T)$ in the ferroelectric phase scales with the temperature dependence of the square root of $I_{2 \omega}$ measured at both PMGs and is expected to be proportional to the spontaneous polarization $P_{s}$. This conclusion will be subjected to verification, see discussion just below.

Figure 5(a) shows the temperature dependences of $I_{2 \omega}$ determined at heating and cooling for PMG-I (solid and open circles) and PMG-II (solid and open squares), respectively. In the following we compare it with the intensity of SHG in 


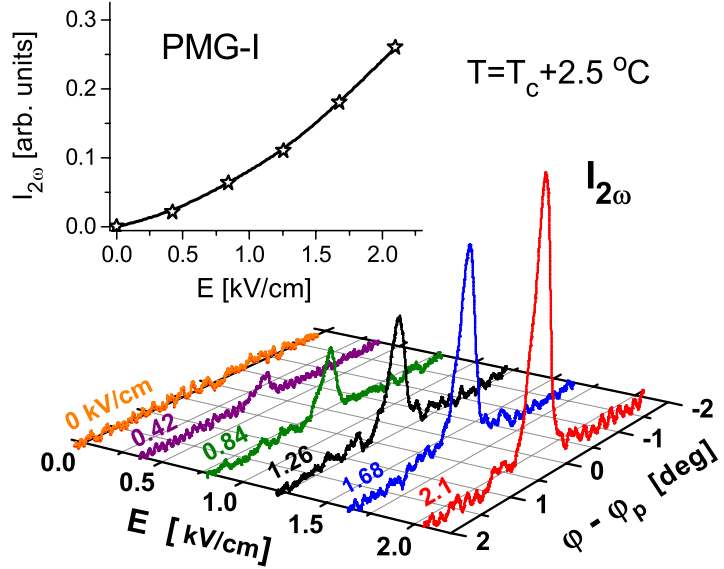

FIG. 6. (Color online) The angular dependencies of the SHG intensity $I_{2 \omega}$ in the vicinity of the phase matching angle $\varphi_{p}$ for PMG-I measured at different applied electric fields in the paraelectric phase $\left(T=T_{c}+2.5^{\circ} \mathrm{C}\right)$. Inset shows the electric field dependence of $I_{2 \omega}$ being extracted at each $E$ as maxima of the presented angular $\varphi$-dependences in the figure.

$\mathrm{SiO}_{2}\left(\chi_{111}\right.$-component), one obtains at $T=23{ }^{\circ} \mathrm{C}$ the magnitudes $\quad \chi_{\text {eff }}^{(2)}(\mathrm{PMG}-\mathrm{I})=4.0 \mathrm{pm} / \mathrm{V} \quad$ and $\quad \chi_{\mathrm{eff}}^{(2)}(\mathrm{PMG}-\mathrm{II})$ $=3.6 \mathrm{pm} / \mathrm{V}$. Figure $5(\mathrm{~b})$ shows the temperature dependences of $\chi_{\text {eff }}^{(2)}$ determined in PMG-I and PMG-II. The ferroelectricto-paraelectric PT is accompanied by a vanishing of SHG, however the variations in $\chi_{\mathrm{eff}}^{(2)}(T)$ in the vicinity of the Curie point appears to be smeared over the temperature range of about $1.5^{\circ} \mathrm{C}$ and are characterized for both PMGs by the temperature hysteresis of about $0.5^{\circ} \mathrm{C}$. A smeared character of this PT may be an evidence for coexistence of metastable polar and nonpolar regions indicating on a sufficiently spatially inhomogeneous structure that occurs in the region of the Curie point $T_{c}$. Using the spontaneous polarization $P_{s}(T)$ measured in Ref. 8 we present in Fig. 5(c) the dependences of $\chi_{\text {eff }}^{(2)}$ versus $P_{s}$. They appears to be linear for both PMGs; $\chi_{\text {eff }}^{(2)}=b P_{s}$, where $b$ is the characteristic effective constant. Linear fitting of the $\chi_{\text {eff }}^{(2)}\left(P_{s}\right)$-dependence allows to derive directly the magnitude of the constant $b$ which appears to be equal to $5.1 \times 10^{-11}$ and $4.6 \times 10^{-11} \mathrm{~m}^{3} / \mathrm{V} \mathrm{C}$ as for PMG-I and PMG-II, respectively. Within such approach the quadratic nonlinearity is considered to be induced by the spontaneous polarization, thus we deal with the spontaneous SHG as has been mentioned above. In the paraelectric phase the SHG does not occur since $P_{s}=0$. On the other hand, this behavior can be also interpreted applying the symmetry principle. According to it the quadratic nonlinearity does not occur in the paraelectric phase because in the centrosymmetric media all the tensor components $\chi_{n j m}^{(2)}$ are equal 0 . The spontaneous polarization $P_{s}$, that appears below $T_{c}$, plays here a role of a symmetry breaking parameter which lowers the symmetry from the centrosymmetric $\mathrm{D}_{2 h}$ to the noncentrosymmetric $\mathrm{C}_{2 v}$ group.

Following this route, the symmetry can be lowered as well due to the induced polarization $P_{\text {ind }}$ caused by an applied external field $E$. In this case $\chi_{\text {eff }}^{(2)}=b P_{\text {ind }}$, where $P_{\text {ind }}$ $=\varepsilon_{0}(\varepsilon-1) E, \varepsilon$ is the static dielectric constant. Figure 6 reports the SHG in the centrosymmetric paraelectric phase of $\mathrm{AgNa}\left(\mathrm{NO}_{2}\right)_{2}$ driven by the electric field being applied along the $Y$-axis. Inset of Fig. 6 demonstrate the field dependence

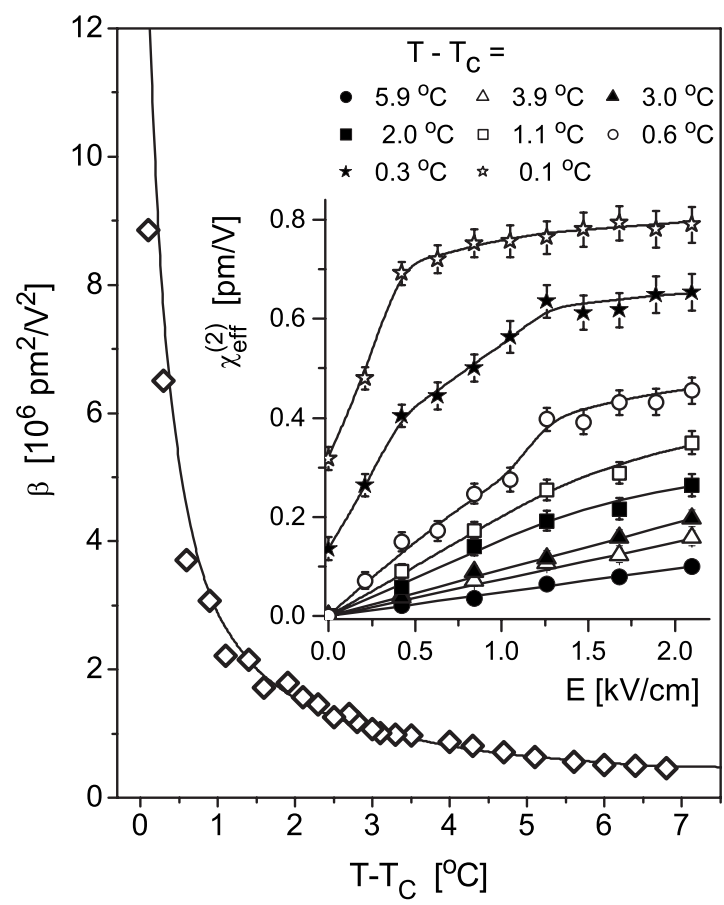

FIG. 7. Temperature dependence of the NLO coefficient $\beta=d \chi_{\text {eff }}^{(2)} / d E$ determined in PMG-I in the paraelectric phase. $\beta$ has been obtained in the region of small electric field $(E \rightarrow 0)$ being applied along the polar $Y$-axis. Inset shows $\chi_{\text {eff }}^{(2)}(E)$-dependences measured at several temperatures above $T_{c}$.

of the $I_{2 \omega}$ being extracted as maxima of $\varphi$-dependences shown in Fig. 6, i.e., at PMG-I geometry $\left(\varphi=\varphi_{p}, \theta=\theta_{p}\right)$. Square root of SHG signal $\left(I_{2 \omega}^{1 / 2}\right)$ is again proportional to $\chi_{\mathrm{eff}}^{(2)}$. Far above $T_{c}$ the $\chi_{\text {eff }}^{(2)}(E)$-dependences are nearly linear, but approaching $T_{c}$ they gradually becomes more nonlinear, see inset in Fig. 7. In the close vicinity of PT-point the $\chi_{\text {eff }}^{(2)}(E)$-dependences get a saturated character reproducing the shape of $P_{\text {ind }}(E)$-dependences being typical for ferroelectric materials in this temperature range. ${ }^{6}$ The constant $\beta$, defining as $d \chi_{\text {eff }}^{(2)} / d E$, describes a cubic nonlinearity and is $\propto d P_{\text {ind }} / d E \propto \varepsilon$. At small electric field the $T$-dependence of dielectric constant $\varepsilon(T)$ follows the Curie-Weiss law $C_{0} /(T$ $\left.-T_{0}\right){ }^{7}$ thus one can expect similar anomalous rising of the NLO constant $\beta$ in the vicinity of ferroelectric Curie point $T_{c}$. Figure 7 demonstrates the temperature dependence of $\beta$, determined from the slopes of $\chi_{\mathrm{eff}}^{(2)}(E)$-dependences at $E$ $\rightarrow 0$. One can realize that $\beta(T)$ behaves in analogical manner as static dielectric constant $\varepsilon(T){ }^{7}$ i.e., anomalously rises by approaching the Curie point $T_{c}$. The $\varepsilon(T)$-dependence can be indeed derived from the $\beta(T)$-dependence assuming that one deals with the small applied fields $E$. On the one hand, this means that $\chi_{\text {eff }}^{(2)}=\beta E$. On the other hand, the second order NLO susceptibility $\chi_{\text {eff }}^{(2)}$ can be expressed through the electric field induced polarization $P_{\text {ind }}$, i.e., $\chi_{\text {eff }}^{(2)}=b P_{\text {ind }}=b \varepsilon_{0}(\varepsilon-1) E$. By comparing these expressions one obtains quite simple relation as follows:

$$
\varepsilon=1+\beta /\left(b \varepsilon_{0}\right) .
$$

Figure 8 shows the static dielectric constant $\varepsilon$ and its inverse magnitude $\varepsilon^{-1}$ versus $T$ being determined in the paraelectric phase by using the $\beta(T)$-dependence, given in Fig. 7, and coupling constant $b=5.1 \times 10^{-11} \mathrm{~m}^{3} / \mathrm{C} \mathrm{V}$, as derived from 


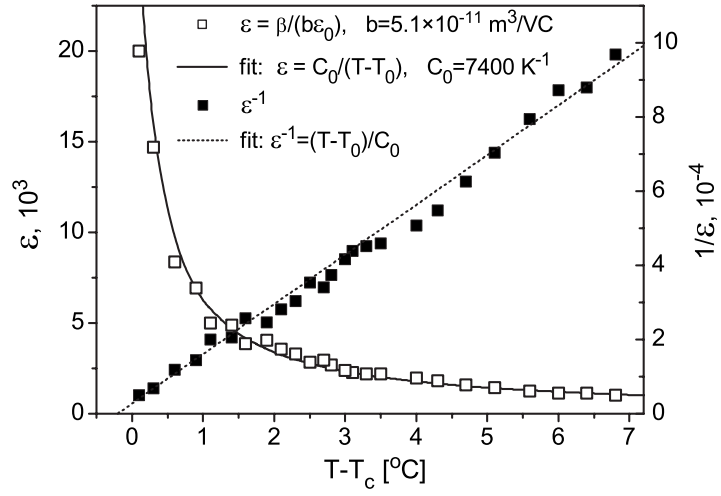

FIG. 8. Static dielectric constant $\varepsilon$ and its inverse magnitude $\varepsilon^{-1}$ vs $T$ being determined in the paraelectric phase from $\beta(T)$-dependence $[\varepsilon(T)$ $\left.=\beta(T) /\left(\varepsilon_{0} b\right)\right]$. Solid lines are the best Curie-Weiss fits providing the magnitude of the Curie-Weiss constant $C_{0}=7400 \mathrm{~K}^{-1}$.

$\chi_{\text {eff }}^{(2)}\left(P_{s}\right)$-dependence [see Fig. 5(c)]. Solid lines are the best Curie-Weiss fits providing the magnitude of the Curie-Weiss constant $C_{0}=7400 \mathrm{~K}^{-1}$ for $\mathrm{AgNa}\left(\mathrm{NO}_{2}\right)_{2}$ crystal. This value is larger comparing to the one $\left(C_{0}=4700 \mathrm{~K}^{-1}\right)$ given in Ref. 7. However, it is very likely that the $C_{0}$ value reported in that work has not been correctly extracted from the presented there $\varepsilon^{-1}(T)$-dependence. Our rough estimation of the data ${ }^{7}$ gives somewhat larger its magnitude, i.e., $C_{0} \approx 5900 \mathrm{~K}^{-1}$. Moreover, by comparing the $C_{0}$ magnitudes obtained by different techniques one must keep in mind different experimental conditions under which the measurements have been performed. In particular, our NLO measurements correspond to a fully static regime $(\omega / 2 \pi=0)$ whereas the conditions in Ref. 7 are quasistatic ones $(\omega / 2 \pi=1000 \mathrm{~Hz})$. This remark appears to be crucial especially for $\mathrm{AgNa}\left(\mathrm{NO}_{2}\right)_{2}$ crystals which are characterized by extremely slow polarization dynamics corresponding to the kilohertz region. ${ }^{9,13,14}$ Due to this reason the difference $\Delta T=T_{c}-T_{0} \approx 2{ }^{\circ} \mathrm{C}$ obtained in (Ref. 7) appears to be substantially larger comparing to the one derived in the present work $\left(\Delta T \approx 0.25{ }^{\circ} \mathrm{C}\right)$ by extrapolating linear dependence $\varepsilon^{-1}(T)$ into the region below $T_{c}$ (see Fig. 8). Really small magnitude of $\Delta T$ can be considered here as an evidence that we indeed deal with the PT of the first order being very close to the tricritical point. ${ }^{10}$

\section{CONCLUSION}

Taken together, we report here on SHG in ferroelectric $\mathrm{AgNa}\left(\mathrm{NO}_{2}\right)_{2}$ crystals being driven by the spontaneous and electric field induced polarizations. Obtained results are interpreted within the phenomenological theory which considers the free energy describing the interaction between the spontaneous or electric field induced polarizations and spatially inhomogeneous electric polarizations resulted from propagating optical waves. At room temperature $\mathrm{AgNa}\left(\mathrm{NO}_{2}\right)_{2}$ crystals are characterized by the effective NLO susceptibilities $\chi_{\text {eff }}^{(2)}$ being comparable or significantly larger comparing to a large number of other known NLO materials such as inorganic crystals $\mathrm{SiO}_{2},{ }^{24} \mathrm{KH}_{2} \mathrm{PO}_{4},{ }^{24} \mathrm{PbHPO}_{4},{ }^{25}$ $\mathrm{BaAlBO}_{3} \mathrm{~F}_{2},{ }^{26} \quad \mathrm{Bi}_{2} \mathrm{ZnOB}_{2} \mathrm{O}_{6}$ (Ref. 27) or organic crystals 3- and 4-aminophenol- $\mathrm{H}_{3} \mathrm{PO}_{4},{ }^{28}$ L-histidinium-Ltartrate hemihydrate ${ }^{29}$ and $\left(\mathrm{H}_{3} \mathrm{~N}\left(\mathrm{CH}_{2}\right)_{2} \mathrm{SS}\left(\mathrm{CH}_{2}\right)_{2} \mathrm{NH}_{3}\right)$
$\mathrm{PbI}_{5} \cdot \mathrm{H}_{2} \mathrm{O} .{ }^{30,31}$ However, it is more important that relatively high magnitudes of the effective NLO susceptibilities are combined in $\mathrm{AgNa}\left(\mathrm{NO}_{2}\right)_{2}$ crystals with several PMGs. This allows to consider them as high-performance materials for potential NLO applications, such as frequency doubling, parametric generation and amplification, or other applications that require a high-efficient frequency conversion. In addition, an anomalously large response of NLO susceptibilities with respect to an applied electric field has been found in the vicinity of the Curie point of $\mathrm{AgNa}\left(\mathrm{NO}_{2}\right)_{2}$ crystals. This may also have a number of applications, especially in those devices where an efficient tunable control of SHG intensity is demanded.

\section{ACKNOWLEDGMENTS}

This work has been supported by grant Moltech-Anjou (Angers, France) likewise by the Program of the UkrainianPolish Scientific-Technical Cooperation in Years 2009-2010 [Project title: "Investigations of new crystalline materials for optoelectronics applications: characterization, geometry optimization, and improvement of their efficiency in practical applications" Project No. M/138-2009 (according to Ukrainian classification); Project No.: 16 (according to Polish classification)].

${ }^{1}$ B. E. A. Saleh and M. C. Teich, Fundamentals of Photonics (Wiley, New York, 1991).

${ }^{2}$ F. Agulló-López, J. M. Cabrera, and F. Agulló-Rueda, Electro-Optics, Phenomena, Materials, and Applications (Academic, New York, 1994).

${ }^{3}$ R. Dhanasekaran, IOP Conf. Ser.: Mater. Sci. Eng. 2, 012014 (2009).

${ }^{4}$ M. DrDomenico, Jr. and S. H. Wemple, J. Appl. Phys. 40, 720 (1969).

${ }^{5}$ A. V. Kityk, R. Czaplicki, A. Klöpperpieper, A. S. Andrushchak, and B. Sahraoui, Appl. Phys. Lett. 96, 061911 (2010).

${ }^{6}$ F. Jona and G. Shirane, Ferroelectric Crystals (Dover, New York, 1993).

${ }^{7}$ K. Gesi, J. Phys. Soc. Jpn. 28, 395 (1970).

${ }^{8}$ K. Gesi, J. Phys. Soc. Jpn. 33, 108 (1972).

${ }^{9}$ J. Petersson, E. Schneider, and R. Siems, Z. Phys. B: Condens. Matter 39, 233 (1980).

${ }^{10}$ J. Helwig, J. Petersson, and E. Schneider, Z. Phys. B 28, 87 (1977).

${ }^{11}$ V. P. Soprunyuk, A. Fuith, H. Kabelka, K. Knorr, A. Klöpperpieper, K. Sokalski, and A. V. Kityk, Phys. Rev. B 66, 104102 (2002).

${ }^{12}$ J. Grossmann, D. Muller, and J. Petersson, Z. Naturforsch. A 29A, 1055 (1974).

${ }^{13}$ J. Grossmann, D. Muller, J. Petersson, and E. Schneider, Z. Naturforsch. A 31A, 1089 (1976).

${ }^{14}$ Y. Tominaga, S. Wada, and S. Iida, J. Phys. Soc. Jpn. 32, 1675 (1972).

${ }^{15}$ R. C. Miller, Appl. Phys. Lett. 5, 17 (1964).

${ }^{16}$ A. V. Kityk, O. M. Mokry, V. P. Soprunyuk, and O. G. Vlokh, J. Phys.: Condens. Matter 5, 5189 (1993).

${ }^{17}$ K. T. Mashiyama and H. Mashiyama, J. Phys. Soc. Jpn. 56, 1810 (1987).

${ }^{18}$ O. G. Vlokh, A. V. Kityk, O. M. Mokry, and V. G. Grybyk, Phys. Status Solidi A 116, 287 (1989).

${ }^{19}$ A. V. Kityk, V. P. Soprunyuk, A. Fuith, W. Schranz, and H. Warhanek, Phys. Rev. B 53, 6337 (1996).

${ }^{20}$ H. Miki, Y. Makita, and K. Gesi, J. Phys. Soc. Jpn. 30, 1512 (1971).

${ }^{21}$ B. Sahraoui, J. Luc, A. Meghea, R. Czaplicki, J.-L. Fillaut, and A. Migalska-Zalas, J. Opt. A, Pure Appl. Opt. 11, 024005 (2009).

${ }^{22}$ P. D. Maker, R. W. Terhue, M. Nisenoff, and C. M. Savage, Phys. Rev. Lett. 8, 21 (1962).

${ }^{23}$ J. Jerphagnon and S. K. Kurtz, J. Appl. Phys. 41, 1667 (1970).

${ }^{24}$ I. Shoji, T. Kondo, A. Kitamoto, M. Shirane, and R. Ito, J. Opt. Soc. Am. B 14, 2268 (1997).

${ }^{25}$ A. Keens and H. Happ, J. Phys. C 21, 1661 (1988).

${ }^{26}$ Z.-G. Hu, M. Yoshimura, K. Muramatsu, Y. Mori, and T. Sasaki, Jpn. J. Appl. Phys., Part 2 41, L1131 (2002).

${ }^{27}$ F. Li, S. Pan, X. Hou, and J. Yao, Cryst. Growth Des. 9, 4092 (2009). 
${ }^{28}$ T. Głowiak, S. Debrus, M. May, A. J. Barnes, and H. Ratajczak, J. Mol. Struct. 596, 77 (2001).

${ }^{29}$ M. K. Marchewka, S. Debrusb, A. Pietraszko, A. J. Barnesc, and H. Ratajczak, J. Mol. Struct. 656, 265 (2003).
${ }^{30}$ W. Bi, N. Louvain, N. Mercier, J. Luc, I. Rau, F. Kajzar, and B. Sahraoui, Adv. Mater. (Weinheim, Ger.) 20, 1013 (2008).

${ }^{31}$ N. Louvain, N. Mercier, J. Luc, and B. Sahraoui, Eur. J. Inorg. Chem. 2008, 3592 . 